\title{
Lessons in Translational Non-Crystallographic Symmetry: Solving the Crystal Structure of a Putative Protease from Gemella haemolyans \\ N Tran' ${ }^{1}$, E Eisenmesser ${ }^{2}$, T Holyoak ${ }^{3}$ \\ ${ }^{1} \mathrm{NA}{ }^{2}$ University of Colorado Anschutz Medical Campus, ${ }^{3}$ Biology Dept, Univ of Waterloo n9tran@uwaterloo.ca
}

The $2.0 \AA$ crystal structure of a putative trypsin-like protease from Gemella haemolyans was solved to help identify its potential substrates and biological function. Despite the availability of a $31 \%$ sequence identity and $78 \%$ coverage search model, the structure was unable to be solved using routine molecular replacement strategies due to the crystal's strong translational non-crystallographic symmetry (tNCS) and large structural differences between several loops found in the N-terminal lobes of the solved structure and search models.

Although a significant portion of the C-terminal lobe was readily resolved by performing molecular replacement with an ensemble search model (MrBUMP), the rest of the structure could not be extrapolated from this partial structure due to the aforementioned factors. Attempts to manually solve for the tNCS and the use of homology modelling to find better search models did not aid in structure solution. The structure was finally solved by Rosettacoupled molecular replacement (phenix.mr_rosetta), which was able to build the rest of the structure ab initio from this partial model. With the final structure confirming that the putative protease has a trypsin-like fold and the required active-site residues, future studies will begin to investigate the protease's biological role. 